Mathematical Modelling and Analysis

Volume 18 Number 1, February 2013, 122-135

http://dx.doi.org/10.3846/13926292.2013.760012

(c) Vilnius Gediminas Technical University, 2013
Publisher: Taylor\&Francis and VGTU

http://www.tandfonline.com/TMMA

Print ISSN: 1392-6292

Online ISSN: 1648-3510

\title{
Basic Theory of Nonlinear Third-Order $q$-Difference Equations and Inclusions
}

\author{
Bashir Ahmad $^{a}$ and Juan Jose Nieto ${ }^{a, b}$ \\ ${ }^{a}$ King Abdulaziz University, Faculty of Science \\ P.O. Box 80203, 21589 Jeddah, Saudi Arabia \\ ${ }^{b}$ Universidad de Santiago de Compostela, Facultad de Matemáticas \\ 15782 Santiago, Spain \\ E-mail: bashir_qau@yahoo.com \\ E-mail(corresp.): juanjose.nieto.roig@usc.es
}

Received June 25, 2012; revised November 30, 2012; published online February 1, 2013

\begin{abstract}
We investigate the existence of solutions for boundary value problems of the third-order $q$-difference equations and inclusions. Our results are based on some standard fixed point theorems. In case of inclusion problem, the existence results are obtained for convex as well as nonconvex multi-valued maps. We also discuss the existence of extremal solutions for the inclusion problem.
\end{abstract}

Keywords: $q$-difference equations, inclusions, fixed point, extremal solution.

AMS Subject Classification: 34A60; 39A13.

\section{Introduction}

The subject of $q$-difference equations, initiated in the beginning of the 20th century $[4,16,32,39]$, has evolved into a multidisciplinary subject, for example, see $[21,22,23,24,25,26,27,34]$ and references therein. For some recent work on $q$-difference equations, we refer the reader to the papers $[3,6,7,8,11,15$, $19,20,31]$. However, the theory of boundary value problems for nonlinear $q$ difference equations is still in the initial stages and many aspects of this theory need to be explored.

Differential inclusions appear in the mathematical modelling of certain problems in economics, optimal control, stochastic analysis, etc., and have recently been studied by many authors, for instance, see $[12,13,29,30,36,37,41]$ and the references therein. Recently, Ahmad and Ntouyas [9] investigated a boundary value problem of the second-order $q$-difference inclusions with nonseparated boundary conditions. For some q-fractional differential equations, see $[1,2,33]$, and for some recent work in q-difference equations with delay we refer to $[10]$. 
We study the existence of solutions for boundary value problems of thirdorder $q$-difference equations given by

$$
D_{q}^{3} u(t)=f(t, u(t)), \quad t \in J,
$$

and inclusions of the form

$$
D_{q}^{3} u(t) \in F(t, u(t)), \quad t \in J
$$

with the boundary conditions

$$
u(0)=0, \quad D_{q} u(0)=0, \quad u(1)=0 .
$$

Here $f: J \times \mathbb{R} \rightarrow \mathbb{R}$ is a given continuous function, $J=\left\{q^{n}: n \in \mathbb{N}\right\} \cup\{0,1\}$, $q \in(0,1)$ is a fixed constant, and $F: J \times \mathbb{R} \rightarrow \mathcal{P}(\mathbb{R})$ is a multi-valued function, with $\mathcal{P}(\mathbb{R})$ being the family of all nonempty subsets of $\mathbb{R}$.

We recall that some existence results for the problem (1.1), (1.3), based on Leray-Schauder degree theory and contraction mapping principle, were obtained in [4]. Here, we discuss the existence of solutions for the problem (1.1)(1.3) when the nonlinear function $f(t, u(t))$ is of Carathéodory type and satisfies a generalized variant of Lipschitz condition. However, in this paper, our main aim is to establish some existence results for the inclusion problem (1.2), (1.3) by applying the concept of lower and upper solutions.

\section{Preliminaries}

Let us recall some basic concepts of $q$-calculus [27, 34].

For $0<q<1$, we define the $q$-derivative of a real-valued function $f$ as

$$
D_{q} f(t)=\frac{f(t)-f(q t)}{(1-q) t}, \quad t \in J-\{0\}, \quad D_{q} f(0)=\lim _{t \rightarrow 0} D_{q} f(t) .
$$

Note that for $f$ differentiable at $t$ we have

$$
\lim _{q \rightarrow 1^{-}} D_{q} f(t)=f^{\prime}(t)
$$

The higher order $q$-derivatives are defined inductively as

$$
D_{q}^{0} f(t)=f(t), \quad D_{q}^{n} f(t)=D_{q} D_{q}^{n-1} f(t), \quad n \in \mathbb{N} .
$$

For example, $D_{q}\left(t^{k}\right)=[k]_{q} t^{k-1}$, where $k$ is a positive integer and the $q$-bracket $[k]_{q}=\left(q^{k}-1\right) /(q-1)$. In particular, $D_{q}\left(t^{2}\right)=(1+q) t$.

For $y \geq 0$, let us set $\mathcal{J}_{y}=\left\{y q^{n}: n \in \mathbb{N} \cup\{0\}\right\} \cup\{0\}$ and define the definite $q$-integral of a function $f: \mathcal{J}_{y} \rightarrow \mathbb{R}$ by

$$
I_{q} f(y)=\int_{0}^{y} f(s) d_{q} s=\sum_{n=0}^{\infty} y(1-q) q^{n} f\left(y q^{n}\right)
$$


provided that the series converges. For $b_{1}, b_{2} \in \mathcal{J}_{y}\left(b_{1}=y q^{n_{1}}, b_{2}=y q^{n_{2}}\right.$ for some $n_{1}, n_{2} \in \mathbb{N}$ ), we define

$$
\int_{b_{1}}^{b_{2}} f(s) d_{q} s=I_{q} f\left(b_{2}\right)-I_{q} f\left(b_{1}\right)=(1-q) \sum_{n=0}^{\infty} q^{n}\left[b_{2} f\left(b_{2} q^{n}\right)-b_{1} f\left(b_{1} q^{n}\right)\right] .
$$

Similarly, we have

$$
I_{q}^{0} f(t)=f(t), \quad I_{q}^{n} f(t)=I_{q} I_{q}^{n-1} f(t), \quad n \in \mathbb{N} .
$$

Observe that $D_{q} I_{q} f(x)=f(x)$, and if $f$ is continuous at $x=0$, then

$$
I_{q} D_{q} f(x)=f(x)-f(0) .
$$

This implies that if $D_{q} f(t)=\sigma(t)$, then $f(t)=I_{q} \sigma(t)+c$, where $c$ is an arbitrary constant.

In $q$-calculus, the product rule and integration by parts formula are

$$
\begin{aligned}
& D_{q}(g h)(t)=D_{q} g(t) h(t)+g(q t) D_{q} h(t) \\
& \int_{0}^{x} f(t) D_{q} g(t) d q t=[f(t) g(t)]_{0}^{x}-\int_{0}^{x} D_{q} f(t) g(q t) d_{q} t .
\end{aligned}
$$

In the limit $q \rightarrow 1^{-}$, the above results correspond to their counterparts in standard calculus.

\section{$3 \quad q$-Difference Equations}

This section deals with the existence of solutions for the problem (1.1)-(1.3).

A mapping $\varrho: J \times \mathbb{R} \rightarrow \mathbb{R}$ is said to be Carathéodory if

(i) $t \mapsto \varrho(t, u)$ is measurable for each $u \in \mathbb{R}$,

(ii) $u \mapsto \varrho(t, u)$ is continuous for $t \in J$.

A Carathéodory function $\varrho$ is called $L^{1}(J, \mathbb{R})$-Carathéodory if there exists a function $h_{r} \in L^{1}(J, \mathbb{R})$ for each $r>0$ such that $|\varrho(t, u)| \leq h_{r}(t), t \in J$ for all $u \in \mathbb{R}$ with $|u| \leq r$.

A Carathéodory function $\varrho$ is called $L_{X}^{1}(J, \mathbb{R})$-Carathéodory if there exists a function $h \in L^{1}(J, \mathbb{R})$ such that $|\varrho(t, u)| \leq h(t) t \in J$ for all $u \in \mathbb{R}$, where $h$ is called the bounding function of $\varrho$.

Using the ideas of [4], we know that the solutions of the problem (1.1)-(1.3) are given by the solutions of the equation

$$
u(t)=\int_{0}^{1} G(t, s ; q) f(s, u(s)) d_{q} s
$$

where $G(t, s ; q)$ is the Green's function given by

$$
G(t, s ; q)=\frac{1}{(1+q)} \begin{cases}q s(1-t)\left[q^{2} s(1+t)-(1+q) t\right], & 0 \leq s<t \leq 1 \\ t^{2}(1-q s)\left(q^{2} s-1\right), & 0 \leq t \leq s \leq 1\end{cases}
$$


We define $\|u\|=\sup \{|u(t)|: t \in J\}$ and set

$$
G_{1}=\max _{t, s \in[0,1]}|G(t, s ; q)| .
$$

Let $L^{1}([0,1], \mathbb{R})$ be the Banach space of measurable functions $u:[0,1] \rightarrow \mathbb{R}$ which are Lebesgue integrable and normed by $\|u\|_{L^{1}}=\int_{0}^{1}|u(t)| d t$.

Theorem 1. Let us assume that

(H1) the function $f$ is $L_{X}^{1}$-Carathéodory with bounding function $h \in L^{1}\left(J, \mathbb{R}^{+}\right)$, that is, $|f(t, u)| \leq h(t)$ a.e. $t \in J$ for all $u \in \mathbb{R}$ such that

$$
\|h\|_{L^{1}}=\int_{0}^{1}|h(t)| d t<\infty .
$$

Then the boundary value problem (1.1)-(1.3) has at least one solution on $J$.

Proof. In view of the solution representation (3.1), we define an operator $\mathcal{P}$ by

$$
(P u)(t)=\int_{0}^{1} G(t, s ; q) f(s, u(s)) d_{q} s .
$$

For $m>0$ we consider the closed ball $B_{m}=\{u \in C(J, \mathbb{R}):\|u\| \leq m\}$ in $C(J, \mathbb{R})$.

Taking $m=G_{1}\|h\|_{L^{1}}$, and using the assumption (H1), for $u \in \mathbb{R}$, we have

$$
\begin{aligned}
|(\mathcal{P} u)(t)| & \leq \int_{0}^{1}|G(t, s ; q)||f(s, u(s))| d_{q} s \\
& \leq \int_{0}^{1}|G(t, s ; q)||h(s)| d_{q} s \leq G_{1}\|h\|_{L^{1}}=m .
\end{aligned}
$$

Hence $\|P u\| \leq m$, which means that $P$ maps $B_{m}$ into itself. In fact, $P$ maps the convex hull of $P\left(B_{m}\right)$ into itself. Since $f$ is bounded on $B_{m}$, therefore, $P\left(B_{m}\right)$ is equicontinuous. Thus, by the Schauder fixed point theorem, it follows that the operator $\mathcal{P}$ has at least one fixed point $u \in C(J, \mathbb{R})$ such that $P u=u$, which implies that the problem (1.1)-(1.3) has at least one solution on $J$. This completes the proof.

Theorem 2. Assume that

(H2) there exists a function $\ell(t) \in L^{1}\left(J, \mathbb{R}^{+}\right)$such that for each $u, v \in C[J, \mathbb{R}]$, we have

$$
|f(t, u)-f(t, v)| \leq \ell(t)|u(t)-v(t)|
$$

Then the problem (1.1)-(1.3) has a unique solution on $J$ if $\|\ell\|_{L^{1}} G_{1}<1$.

Proof. By the assumption (H2) together with (3.3), we have

$$
\begin{aligned}
|(\mathcal{P} u)(t)-(\mathcal{P} v)(t)| & \leq \int_{0}^{1}|G(t, s ; q)||f(s, u(s))-f(s, v(s))| d_{q} \\
& \leq\|u-v\| \int_{0}^{1}|G(t, s ; q)| \ell(s) d_{q} s \leq\|\ell\|_{L^{1}} G_{1}\|u-v\|,
\end{aligned}
$$


which implies that $\mathcal{P}$ is a contraction mapping as $\|\ell\|_{L^{1}} G_{1}<1$. Thus, by Banach fixed point theorem, there exists a unique solution for the problem (1.1)-(1.3). This completes the proof.

\section{$4 \quad q$-Difference Inclusions}

We begin this section with some basic concepts of multi-valued maps $[12,13$, 29, 30, 41].

For a normed space $(X,\|\|$.$) , let \mathcal{P}_{c l}(X)=\{Y \in \mathcal{P}(X): Y$ is closed $\}$, $\mathcal{P}_{b}(X)=\{Y \in \mathcal{P}(X): Y$ is bounded $\}, \mathcal{P}_{c p}(X)=\{Y \in \mathcal{P}(X): Y$ is compact $\}$, $\mathcal{P}_{c l, c}(X)=\{Y \in \mathcal{P}(X): Y$ is closed and convex $\}, \mathcal{P}_{c l, b}(X)=\{Y \in \mathcal{P}(X)$ : $Y$ is closed and bounded $\}$, and

$$
\mathcal{P}_{c p, c}(X)=\{Y \in \mathcal{P}(X): Y \text { is compact and convex }\} .
$$

A multi-valued map $\mathcal{G}: X \rightarrow \mathcal{P}(X)$ is convex (closed) valued if $\mathcal{G}(x)$ is convex (closed) for all $x \in X$. The map $\mathcal{G}$ is bounded on bounded sets if $\mathcal{G}(\mathbb{B})=\bigcup_{x \in \mathbb{B}} \mathcal{G}(x)$ is bounded in $X$ for all $\mathbb{B} \in \mathcal{P}_{b}(X)$ (i.e. $\sup _{x \in \mathbb{B}}\{\sup \{|y|: y \in$ $\mathcal{G}(x)\}\}<\infty)$. $\mathcal{G}$ is called upper semi-continuous (u.s.c) on $X$ if for each $x_{0} \in X$, the set $\mathcal{G}\left(x_{0}\right)$ is nonempty closed subset of $X$ and if for each open set $N$ of $X$ containing $G\left(x_{0}\right)$, there exists an open neighbourhood $N_{0}$ of $x_{0}$ such that $\mathcal{G}\left(N_{0}\right) \subseteq N$. In other words $\mathcal{G}$ is u.s.c if the set $\mathcal{G}^{-1}(A)=\{x \in X: \mathcal{G} \subset A\}$ is open in $X$ for every open set $A$ in $X$. $\mathcal{G}$ is called lower semi-continuous (l.s.c.) on $X$ if $A$ is any open subset of $X$ then $\{x \in X: \mathcal{G} x \cap A \emptyset\}$ is open in $X$. $\mathcal{G}$ is called continuous if it is lower as well as upper semi-continuous on $X$. $\mathcal{G}$ is called compact if for every $M$ bounded subset of $X, \mathcal{G}(M)$ is relatively compact. Finally $\mathcal{G}$ is called completely continuous if it is upper semi-continuous and compact on $X$.

A multivalued map $\mathcal{G}:[0 ; 1] \rightarrow P_{c l}(\mathbb{R})$ is said to be measurable if for every $y \in \mathbb{R}$, the function

$$
t \longmapsto d(y, \mathcal{G}(t))=\inf \{|y-z|: z \in \mathcal{G}(t)\}
$$

is measurable.

Let $C([0,1])$ denote a Banach space of continuous functions from $[0,1]$ into $\mathbb{R}$ with the norm defined by $\|u\|=\sup \{|u(t)|: t \in J\}$ for each $u \in C([0,1])$.

Definition 1. A multivalued map $F: J \times \mathbb{R} \rightarrow \mathcal{P}(\mathbb{R})$ is said to be Carathéodory if

(i) $t \longmapsto F(t, x)$ is measurable for each $x \in \mathbb{R}$;

(ii) $x \longmapsto F(t, x)$ is upper semicontinuous for $t \in J$;

Further a Carathéodory function $F$ is called $L^{1}$-Carathéodory if

(iii) for each $\alpha>0$, there exists $\varphi_{\alpha} \in L^{1}\left([0, T], \mathbb{R}^{+}\right)$such that

$$
\|F(t, x)\|=\sup \{|v|: v \in F(t, x)\} \leq \varphi_{\alpha}(t)
$$

for all $\|x\| \leq \alpha$ and for $t \in J$. 
Definition 2. A multivalued function $F: J \times \mathbb{R} \rightarrow \mathcal{P}(\mathbb{R})$ is called $L_{X^{-}}^{1}$ Carathéodory if there exists a function $h \in L^{1}(J, \mathbb{R})$ such that

$$
\|F(t, u)\|=\sup \{|f|: f \in F(t, u)\} \leq h(t), \quad \text { a.e. } t \in J
$$

for all $x \in \mathbb{R}$, and the function $h$ is called a bounding function of $F$ on $J \times \mathbb{R}$.

Let $A, B \in \mathcal{P}_{c l}(X)$, let $a \in A$ and let

$$
D(a, B)=\inf \{\|a-b\|: b \in B\} \quad \text { and } \quad D_{1}(A, B)=\sup \{D(a, B): a \in A\} .
$$

The function $H: \mathcal{P}_{c l}(X) \times \mathcal{P}_{c l, b}(X) \rightarrow[0,+\infty)$ defined by

$$
H(A, B)=\max \left\{D_{1}(A, B), D_{1}(B, A)\right\}
$$

is a metric and is called Hausdorff metric on $X$. Notice that $\left(\mathcal{P}_{c l, b}(X), H\right)$ is a metric space, $\left(\mathcal{P}_{c l}(X), H\right)$ is a complete metric space [35] and

$$
H(\emptyset, C)=\sup \{\|c\|: c \in C\}, \quad C \in \mathcal{P}_{b}(X) .
$$

Definition 3. A multi-valued function $F: \mathbb{R} \rightarrow \mathcal{P}_{c l}(\mathbb{R})$ is called

(i) $\gamma$-Lipschitz if there exists $\gamma>0$ (Lipschitz constant) such that

$$
H(F(x), F(y)) \leq \gamma\|x-y\|, \quad \text { for each } x, y \in X
$$

(ii) a contraction if it is $\gamma$-Lipschitz with $\gamma<1$.

Definition 4. A multi-valued function $F: J \times \mathbb{R} \rightarrow \mathcal{P}_{c l}(\mathbb{R})$ is called

(i) $\gamma(t)$-Lipschitz if there exists $\gamma \in L^{1}\left(J, \mathbb{R}^{+}\right)$such that

$$
H(F(t, x), F(t, y)) \leq \gamma(t)\|x-y\|, \quad \text { for each } x, y \in X
$$

(ii) a contraction if it is $\gamma(t)$-Lipschitz with $\|\gamma\|<1$.

For the forthcoming analysis, we need the following lemmas.

Lemma 1. [14] Let $M \subset X$. If $F: M \rightarrow \mathcal{P}(X)$ is closed and $F(M)$ is relatively compact then $F$ is u.s.c. on $M$. Moreover, if $F: X \rightarrow \mathcal{P}(X)$ is closed valued and compact function, then $F$ is u.s.c.on $X$.

Lemma 2. [38] Let $T: X \rightarrow \mathcal{P}_{c p, c}(X)$ be a completely continuous multi-valued function. If

$$
\mathcal{E}=\{u \in X: \lambda u \in T u, \text { for some } \lambda>1\}
$$

is a bounded set, then $T$ has a fixed point.

Lemma 3. [18] Let $(X, d)$ be a complete metric space. If $\mathcal{G}: X \rightarrow \mathcal{P}_{c l}(X)$ is a contraction in the sense of Definition $4(\mathrm{ii})$, then $\mathcal{G}$ has a fixed point. 
Now we are in a position to discuss the existence of solutions for the problem (1.2)-(1.3) when the right hand side is convex as well as non-convex valued.

Let us define the set of selections of $F$ as

$$
S_{F}(u)=\left\{f \in L^{1}(J, \mathbb{R}): f(t) \in F(t, u(t)) \text { a.e. } t \in J\right\} .
$$

Theorem 3. Let us assume that

(H3) the multi-valued function $F: J \times \mathbb{R} \rightarrow \mathcal{P}_{c l, c}(\mathbb{R})$ is $L_{X}^{1}$-Carathéodory with a growth function $h \in L^{1}\left(J, \mathbb{R}^{+}\right)$, that is, $\|F(t, u)\| \leq h(t)$ a.e. $t \in J$ for all $u \in \mathbb{R}$ such that $\|h\|_{L^{1}}<\infty$.

If $F$ is lower semi-continuous (l.s.c.), then the problem (1.2)-(1.3) has at least one solution on $J$.

Proof. Note that the inclusion problem (1.2)-(1.3) is equivalent to the integral inclusion

$$
u(t) \in \int_{0}^{1} G(t, s ; q) F(s, u(s)) d_{q} s, \quad t \in J,
$$

where $G(t, s ; q)$ is given by $(3.2)$. For each $u$ in $\mathbb{R}$, the set $S_{F}(u)$ is nonempty as $F$ has a non-empty measurable selection by (H3) [17]. Thus there exists a function $f \in F$ such that $f$ is a $L_{X}^{1}$-Carathéodory function with a bounded function $h \in L^{1}\left(J, \mathbb{R}^{+}\right)$and $\|f(t, u)\| \leq\|h(t)\|$ a.e. $t \in J$ for all $u \in \mathbb{R}$. Hence the assumption of Theorem 1 is satisfied and consequently the inclusion (4.1) has a solution, which implies that here exists at least one solution for (1.2)(1.3). This completes the proof.

Let $W^{n, 1}(J, \mathbb{R})$ denotes the Sobolev class of functions $u: J \rightarrow \mathbb{R}$ for which $u^{(n-1)}$ are absolutely continuous and $u^{(n)} \in L^{1}(J, \mathbb{R})$. We define the partial ordering $\leq$ in $W^{n, 1}(J, \mathbb{R})$ as follows: for $u, v \in W^{n, 1}(J, \mathbb{R})$, we define

$$
u \leq v \quad \Leftrightarrow \quad u(t) \leq v(t), \quad \text { for all } t \in J
$$

If $a, b \in W^{n, 1}(J, \mathbb{R})$ with $a \leq b$, then an ordered interval $[a, b] \in W^{n, 1}(J, \mathbb{R})$ is defined by

$$
[a, b]:=\left\{u \in W^{n, 1}(J, \mathbb{R}): a \leq u \leq b\right\} .
$$

Definition 5. A function $\sigma$ is called a lower solution of (1.2)-(1.3) if there exists an $L^{1}(J, \mathbb{R})$ function $f_{1}(t)$ in $F(t, \sigma(t))$ a.e. $t \in J$ such that

$$
D_{q}^{3} \sigma(t) \leq f_{1}(t), \quad \text { a.e. } t \in J, \quad \sigma(0) \leq 0, \quad D_{q} \sigma(0) \leq 0, \quad \sigma(1) \leq 0 .
$$

Similarly a function $\rho$ is called an upper solution of the problem (1.2)-(1.3) if there exists an $L^{1}(J, \mathbb{R})$ function $f_{2}(t)$ in $F(t, \rho(t))$, a.e. $t \in J$ such that

$$
D_{q}^{3} \rho(t) \geq f_{2}(t), \quad \text { a.e. } t \in J, \quad \rho(0) \geq 0, \quad D_{q} \rho(0) \geq 0, \quad \rho(1) \geq 0 .
$$

Theorem 4 [Convex case]. Let the assumption (H3) and the following condition hold: 
(H4) the problem (1.2)-(1.3) has a

lower solution $\sigma$ and an upper solution $\rho$ such that $\sigma \leq \rho$ on $J$.

Then the problem (1.2)-(1.3) has at least one solution $u(t)$ such that

$$
\sigma(t) \leq u(t) \leq \rho(t), \quad \text { for all } t \in J
$$

Proof. We shall show that the assumptions of Lemma 2 are satisfied in a suitable Banach space. For that, let us consider the problem

$$
D_{q}^{3} u(t) \in F(t, A u(t)), \quad t \in J, \quad u(0)=0, \quad D_{q} u(0)=0, \quad u(1)=0,
$$

where $A: C(J, \mathbb{R}) \rightarrow C(J, \mathbb{R})$ is the truncation operator defined by

$$
(A u)(t)= \begin{cases}\sigma(t) & \text { if } u(t)<\sigma(t) \\ u(t) & \text { if } \sigma(t) \leq u(t) \leq \rho(t) \\ \rho(t) & \text { if } \rho(t)<u(t)\end{cases}
$$

Thus, the problem for proving the existence of a solution for (1.2)-(1.3) transforms to finding a solution to the integral inclusion

$$
u(t) \in \int_{0}^{1} G(t, s ; q) F(s, A u(s)) d_{q} s, \quad t \in J .
$$

We will study (4.2) in the space of continuous real-valued functions on $J$ endowed with a supremum norm. Let us define an operator $\mathcal{T}: C(J, \mathbb{R}) \rightarrow$ $\mathcal{P}(C(J, \mathbb{R}))$ by

$$
\mathcal{T} u=\left\{u \in C(J, \mathbb{R}): u(t) \in \int_{0}^{1} G(t, s ; q) f(s) d_{q} s, f \in \bar{S}_{F}(A u)\right\}
$$

where

$$
\begin{gathered}
\bar{S}_{F}(A u)=\left\{f \in S_{F}(A u): f(t) \geq \sigma(t) \text { a.e. } t \in \mathcal{A}_{1} \text { and } f(t) \leq \rho(t) \text { a.e. } t \in \mathcal{A}_{2}\right\}, \\
\mathcal{A}_{1}=\{t \in J: u(t)<\sigma(t) \leq \rho(t)\}, \quad \mathcal{A}_{2}=\{t \in J: \sigma(t) \leq \rho(t)<u(t)\} .
\end{gathered}
$$

By (H3), $F$ is measurable and has a nonempty closed selection set $\bar{S}_{F}(u)$ of $S_{F}(u)$ [17]. Now we show that the operator $\mathcal{T}$ satisfies the conditions of Lemma 2. The proof consists of several steps.

Step I. $\mathcal{T}(u)$ is a convex subset of $C(J, \mathbb{R})$. Let $u_{1}, u_{2} \in \mathcal{T}(u)$. Then there exist $f_{1}, f_{2} \in \bar{S}_{F}(u)$ such that

$$
u_{i}(t)=\int_{0}^{1} G(t, s ; q) f_{i}(s) d_{q} s, \quad i=1,2 .
$$

Since $F(t, u)$ has convex values, therefore, for $0 \leq \theta \leq 1$, we obtain

$$
\begin{aligned}
{\left[\theta u_{1}+(1-\theta) u_{2}\right](t) } & =\theta \int_{0}^{1} G(t, s ; q) f_{1}(s) d_{q} s+(1-\theta) \int_{0}^{1} G(t, s ; q) f_{2}(s) d_{q} s \\
& =\int_{0}^{1} G(t, s ; q)\left[\theta f_{1}+(1-\theta) f_{2}\right](s) d_{q} s .
\end{aligned}
$$


Hence, $\left.\theta u_{1}+(1-\theta) u_{2}\right] \in \mathcal{T} u$ and consequently $\mathcal{T}$ has a convex values in $C(J, \mathbb{R})$.

Step II. $\mathcal{T}$ maps bounded sets into bounded sets in $C(J, \mathbb{R})$. Let $B$ be a bounded set in $C(J, \mathbb{R})$. Then there exists a real number $m>0$ such that $\|u\| \leq m$, for all $u \in B$. Now for each $u \in \mathcal{T}$, there exists $f \in \bar{S}_{F}(u)$ such that

$$
u(t)=\int_{0}^{1} G(t, s ; q) f(s) d_{q} s
$$

and, for each $t \in J$, we have

$$
|u(t)| \leq \int_{0}^{1}|G(t, s ; q)||f(s)| d_{q} s \leq G_{1} \int_{0}^{1}|h(s)| d_{q} s=G_{1}\|h\|_{L^{1}} .
$$

This implies that $\mathcal{T}(B)$ is bounded with $\|u\| \leq G_{1}\|h\|_{L^{1}}=m$.

Step III. $\mathcal{T}$ maps bounded sets into equicontinuous sets in $C(J, \mathbb{R})$. For any $t_{1}, t_{2} \in J$ with $\left|t_{1}-t_{2}\right| \leq \delta, \delta>0$, we have

$$
\begin{aligned}
\left|u\left(t_{1}\right)-u\left(t_{2}\right)\right| & \leq \int_{0}^{1}\left|G\left(t_{2}, s ; q\right)-G\left(t_{1}, s ; q\right)\right||f(s)| d_{q} s \\
& \leq \int_{0}^{1}\left|G\left(t_{2}, s ; q\right)-G\left(t_{1}, s ; q\right)\right||h(s)| d_{q} s
\end{aligned}
$$

which is independent of $u$ in a bounded set and the right hand side tends to zero as $t_{2}-t_{1} \rightarrow 0$. So $\mathcal{T}(B)$ is a equicontinuous set.

Step IV. $\mathcal{T}$ is u.s.c. By the Arzela-Ascoli theorem, the set $\mathcal{T}(B)$ is relatively compact. Therefore, $\mathcal{T}$ is a compact operator. So, by Lemma 1 , we have that $\mathcal{T}$ is u.s.c.

Step V. Now we show that the following set is bounded

$$
\mathcal{E}=\{u \in C(J, \mathbb{R}): \lambda u \in \mathcal{T} u \text { for some } \lambda>1\} .
$$

For $u \in \mathcal{E}$, there exists a $f \in \bar{S}_{F}(u)$ such that

$$
|u(t)| \leq \lambda^{-1} \int_{0}^{1} G(t, s ; q)|f(s)| d_{q} s \leq \int_{0}^{1} G(t, s ; q) h(s) d_{q} s \leq G_{1}\|h\|_{L^{1}} .
$$

Hence the set $\mathcal{E}$ is bounded. Consequently, Lemma 2 applies and the operator $\mathcal{T}$ has a fixed point which is a solution for the truncation operator $A$. Next, we show that $u$ is a solution for the problem (1.2)-(1.3). First we show that $u \in[\sigma, \rho]$. If it is not so, then either $\sigma \not \leq u$ or $u \not \leq \rho$ on $\bar{J} \subset J$. If $\sigma \not \leq u$ then for $t_{1}<t_{2}$, we have $\sigma(t)>u(t)$ for all $t$ in $\left(t_{1}, t_{2}\right) \cap J$. Since $\sigma$ is the lower solution of the problem, therefore, for $f \in \bar{S}_{F}(u)$, we have

$$
u(t)=\int_{0}^{1} G(t, s ; q) f(s) d_{q} s \geq \int_{0}^{1} G(t, s ; q) \sigma(s) d_{q} s=\sigma(t)
$$

for all $t \in\left(t_{1}, t_{2}\right)$, which is a contradiction. Similarly, for $u \not \leq \rho$, we obtain a contradiction. Hence $\sigma(t) \leq u(t) \leq \rho(t)$, for all $t \in J$. In consequence, the problem (1.2)-(1.3) has a solution $u \in[\sigma, \rho]$. This completes the proof. 
Theorem 5 [Non-convex case]. Assume that

$(\mathrm{H} 5) F: J \times \mathbb{R} \rightarrow \mathcal{P}_{c l}(\mathbb{R}),(t,.) \mapsto F(t, u)$ is measurable for each $u \in \mathbb{R}$.

(H6) $F: J \times \mathbb{R} \rightarrow \mathcal{P}_{c l}(\mathbb{R})$ is $\ell(t)$-Lipschitz; that is, $H(F(t, u), F(t, v)) \leq$ $\ell(t)\|u-v\|$.

If $G_{1}\|\ell\|_{L^{1}}<1$, then the problem (1.2)-(1.3) has at least one solution on $J$.

Proof. For each $u$ in $\mathbb{R}, F$ has a nonempty measurable selection by the condition (H5). Therefore, the set $S_{F}(u)$ is nonempty [17] and there exists a function $f \in F$, which is $\ell(t)-$ Lipschitz by the assumption (H6). Thus, the conclusion of Theorem 2 implies and the problem (1.2)-(1.3) has a solution. This completes the proof.

Theorem 6 [Non-convex case]. Let the assumptions (H4), (H5), (H6) hold. Then the problem (1.2)-(1.3) has at least one solution $u(t)$ on $J$ such that $\sigma(t) \leq u(t) \leq \rho(t)$ for all $t \in J$ if $G_{1}\|\ell\|_{L^{1}}<1$.

Proof. First, we show that $\mathcal{T}(u) \subset \mathcal{P}_{c l}(\mathcal{C})$ for each $u \in \mathcal{C}=C(J, \mathbb{R})$, where the operator $\mathcal{T}$ is defined by (4.3). Let $\left\{u_{m}\right\}_{m \geq 0} \in \mathcal{T}(u)$ such that $u_{m} \rightarrow \bar{u}$ in $\mathcal{C}$. Then $\bar{u} \in \mathcal{C}$ and there exists $f_{m} \in S_{F}(u)$ such that

$$
u_{m}(t)=\int_{0}^{1} G(t, s ; q) f_{m}(s) d_{q} s, \quad t \in J .
$$

In view of the fact that $F$ has closed values, we have that $f_{m}$ converges to $f$ in $L^{1}(J, \mathbb{R})$ and hence $f \in S_{F}(u)$. Then, for each $t \in J$,

$$
u_{m}(t) \rightarrow \bar{u}(t)=\int_{0}^{1} G(t, s ; q) f(s, u(s)) d_{q} s .
$$

So $\bar{u} \in \mathcal{T}(u)$.

Next, we show that there exists $\gamma<1$ such that

$$
H\left(\mathcal{T}\left(u_{1}\right), \mathcal{T}\left(u_{2}\right)\right) \leq \gamma\|u-v\|_{\mathcal{C}} \quad \text { for each } u_{1}, u_{2} \in \mathcal{C} .
$$

For $u_{1}, u_{2} \in \mathcal{C}$, there exists $f \in F$ by (H6) such that

$$
\left|f\left(t, u_{1}\right)-f\left(t, u_{2}\right)\right| \leq \ell(t)\left\|u_{1}-u_{2}\right\|_{\mathcal{C}} .
$$

Then for $h_{i}(t) \in \mathcal{T}(u), i=1,2$, we have

$$
h_{1}(t)=\int_{0}^{1} G(t, s ; q) f\left(s, u_{1}(s)\right) d_{q} s, \quad h_{2}(t)=\int_{0}^{1} G(t, s ; q) f\left(s, u_{2}(s)\right) d_{q} s .
$$

Thus,

$$
\begin{aligned}
\left|h_{1}(t)-h_{2}(t)\right| & \leq \int_{0}^{1}|G(t, s ; q)|\left|f\left(s, u_{1}(s)\right)-f\left(s, u_{2}(s)\right)\right| d_{q} s \\
& \leq\left\|u_{1}-u_{2}\right\|_{\mathcal{C}} \int_{0}^{1}|G(t, s ; q)|\|\ell\|(s) d_{q} s \\
& \leq G_{1}\|\ell\|_{L^{1}}\|u-v\|_{\mathcal{C}} .
\end{aligned}
$$


Letting $\gamma:=G_{1}\|\ell\|_{L^{1}}$, it follows that

$$
H(\mathcal{T}(u), \mathcal{T}(v)) \leq \gamma\|u-v\|_{\mathcal{C}}, \quad \text { for each } u, v \in \mathcal{C},
$$

where $\gamma<1$. Hence the operator $\mathcal{T}$ is a contraction and has a fixed point by Lemma 3, which corresponds to a solution of the problem (1.2)-(1.3). As in the proof of Theorem 4, it can be shown that the problem (1.2)-(1.3) has a solution $u \in[\sigma, \rho]$. This completes the proof.

\section{$5 \quad$ Extremal Solutions}

This section deals with the existence of extremal solutions for problem (1.2)(1.3) on an ordered Banach space.

Let us introduce a cone $K$ in $C(J, \mathbb{R})$ as

$$
K=\{u \in C(J, \mathbb{R}): u(t) \geq 0, \forall t \in J\}
$$

We define an order relation $\leq$ in $C(J, \mathbb{R})$ as follows: $u \leq v$ if and only if $u(t) \leq v(t) \forall t \in J$. It is known that $K$ is normal in $C(J, \mathbb{R})$ (see [28]). Let $A, B \in \mathcal{P}(X)$. Then, by $A \leq B$, we mean $a \leq b$ for all $a \in A$ and $b \in B$. Thus if $A \leq A$ then it follows that $A$ is a singleton set.

Definition 6. Let $X$ be an ordered Banach space. A mapping $T: X \rightarrow \mathcal{P}(X)$ is called isotone increasing if $x, y \in X$ with $x<y$, we have that $T(x) \leq T(y)$.

Definition 7. A solution $u_{M}(t)$ of $(1.2)-(1.3)$ is called a maximal solution if for every solution $u(t)$ of $(1.1)$, we have $u(t) \leq u_{M}(t)$ for all $t \in J$. A solution $u_{m}(t)$ of (1.2)-(1.3) is said to be minimal solution if $u_{m}(t) \leq u(t)$ for all $t \in J$ where $u(t)$ is any solution of (1.2)-(1.3).

We need the following result of [5] for the sequel. It is a multivalued version of the seminal work of Tarski [42] and the abstract monotone method [40].

Lemma 4. [5] Let $[\sigma, \rho]$ be an order interval in a Banach space and let $T$ : $[\sigma, \rho] \rightarrow \mathcal{P}([\sigma, \rho])$ be a completely continuous and isotone increasing multivalued map. Further if the cone $K$ in $X$ is normal, then $T$ has a least $u_{*}$ and a greatest fixed point $v^{*}$ in $[\sigma, \rho]$. Moreover, the sequences $\left\{u_{n}\right\}$ and $\left\{v_{n}\right\}$ defined by $u_{n+1} \in T u_{n}, u_{0}=\sigma$ and $v_{n+1} \in T v_{n}, v_{0}=\rho$, converge to $u_{*}$ and $v^{*}$ respectively.

Theorem 7. Let (H4) and the following assumptions hold:

(H7) The multi-valued function $F: J \times \mathbb{R} \rightarrow \mathcal{P}(\mathbb{R})$ is Carathéodory.

(H8) $F(t, u(t))$ is nondecreasing in $u$ a.e. $t \in J$; that is, if $u<v$, then $F(t, u) \leq$ $F(t, v)$ a.e. $t \in J$.

Then (1.2)-(1.3) has a minimal and a maximal solution on $J$. 
Proof. We define an operator $\mathcal{K}: C(J, \mathbb{R}) \rightarrow \mathcal{P}(C(J, \mathbb{R}))$ as

$$
\mathcal{K} u=\left\{v \in C(J, \mathbb{R}): v(t)=\int_{0}^{1} G(t, s ; q) f(s) d_{q} s, f \in S_{F}(u)\right\},
$$

and show that $\mathcal{K}$ satisfies the conditions of Lemma 4 . As in the proof of Theorem 4 , it can be shown that $\mathcal{K}$ is completely continuous operator on $[\sigma, \rho]$. Next, we show that $\mathcal{K}$ is isotone increasing on $C(J, \mathbb{R})$. Let $u, v \in C(J, \mathbb{R})$ be such that $u<v$. Let $\sigma \in \mathcal{K} u$ be arbitrary. Then there is a function $f_{1} \in S_{F}(u)$ such that

$$
\sigma(t)=\int_{0}^{1} G(t, s ; q) f_{1}(s) d_{q} s .
$$

Since $F$ is nondecreasing in $u$, therefore, $S_{F}(u) \leq S_{F}(v)$. Consequently, for any $f_{2} \in S_{F}(v)$, we have

$$
\sigma(t) \leq \int_{0}^{1} G(t, s ; q) f_{2}(s) d_{q} s=\rho
$$

for all $t \in J$ and $\rho \in \mathcal{K} v$. This shows that the multi-valued operator $\mathcal{K}$ is isotone increasing on $C(J, \mathbb{R})$; in particular in the set $[\sigma, \rho]$. Since $\sigma$ and $\rho$ are lower and upper solutions of the problem (1.2)-(1.3) on $J$, we have

$$
\sigma(t) \leq \int_{0}^{1} G(t, s ; q) f(s) d_{q} s
$$

for all $f \in S_{F}(\sigma)$ and so $\sigma \leq \mathcal{K} \sigma$. Similarly, it can be shown that $\rho \geq \mathcal{K} \rho$. Hence we have $\sigma \leq \mathcal{K} \sigma \leq \mathcal{K} \rho \leq \rho$. As $\mathcal{K}$ satisfies all the conditions of Lemma 4 , therefore $\mathcal{K}$ has the least and greatest fixed point in $[\sigma, \rho]$. This implies that problem (1.2)-(1.3) has a minimal and a maximal solution on $J$. This completes the proof.

\section{References}

[1] T. Abdeljawad and D. Baleanu. Caputo $q$-fractional initial value problems and a $q$-analogue Mittag-Leffler function. Commun. Nonlinear Sci. Numer. Simul., 16:4682-4688, 2011. http://dx.doi.org/10.1016/j.cnsns.2011.01.026.

[2] T. Abdeljawad, B. Benli and D. Baleanu. A generalized $q$-Mittag-Leffler function by $q$-Captuo fractional linear equations. Abstr. Appl. Anal., p. 11 pages, 2012. http://dx.doi.org/10.1155/2012/546062. Art. No. 546062

[3] N. Abrashina-Zhadaeva and N. Romanova. Algorithms for numerical solving of 2D anomalous diffusion problems. Math. Model. Anal., 17:447-455, 2012. http://dx.doi.org/10.3846/13926292.2012.686123.

[4] C.R. Adams. On the linear ordinary q-difference equation. Ann. Math., 30:195205, 1928. http://dx.doi.org/10.2307/1968274.

[5] R. Agarwal, B. Dhage and D. O'Regan. The upper and lower solution method for differential inclusions via a lattice fixed point theorem. Dynam. Systems Appl., 12:1-7, 2003. 
[6] B. Ahmad. Boundary value problems for nonlinear third-order q-difference equations. Electron. J. Differential Equations, 94:1-7, 2011.

[7] B. Ahmad and J.J. Nieto. On nonlocal boundary value problems of nonlinear $q$-difference equations. Adv. Difference Equ., 81:10 pages, 2012.

[8] B. Ahmad, J.J. Nieto and J. Pimentel. Some boundary value problems of fractional differential equations and inclusions. Comput. Math. Appl., 62:1238-1250, 2011. http://dx.doi.org/10.1016/j.camwa.2011.02.035.

[9] B. Ahmad and S.K. Ntouyas. Boundary value problems for $q$-difference inclusions. Abstr. Appl. Anal., p. 15 pages, 2011.

http://dx.doi.org/10.1155/2011/292860. Article ID 292860

[10] J.O. Alzabut and T. Abdeljawad. Perron's theorem for q-delay difference equations. Appl. Math. Information Sci., 5:74-84, 2011.

[11] M.H. Annaby and Z.S. Mansour. q-Taylor and interpolation series for Jackson q-difference operators. J. Math. Anal. Appl., 344:472-483, 2008. http://dx.doi.org/10.1016/j.jmaa.2008.02.033.

[12] J.P. Aubin and A. Cellina. Differential Inclusions. Springer-Verlag, BerlinHeidelberg, New York, 1984.

[13] J.P. Aubin and H. Frankowska. Set-Valued Analysis. Birkhäuser, Boston, 1990.

[14] C. Avramescu. A fixed point theorem for multivalued mappings. Electron. J. Qual. Theory Differ. Equ., 17:1-10, 2004.

[15] G. Bangerezako. Variational q-calculus. J. Math. Anal. Appl., 289:650-665, 2004. http://dx.doi.org/10.1016/j.jmaa.2003.09.004.

[16] R.D. Carmichael. The general theory of linear $q$-difference equations. Amer. J. Math., 34:147-168, 1912. http://dx.doi.org/10.2307/2369887.

[17] C. Castaing and M. Valadier. Convex Analysis and Measurable Multifunctions, volume 580 of Lecture Notes in Math. Springer-Verlag, Berlin, Heidelberg, New York, 1977.

[18] K. Deimling. Multivalued Differential Equations. Walter De Gruyter, Berlin, New York, 1992.

[19] A. Dobrogowska and A. Odzijewicz. Second order $q$-difference equations solvable by factorization method. J. Comput. Appl. Math., 193:319-346, 2006. http://dx.doi.org/10.1016/j.cam.2005.06.009.

[20] M. El-Shahed and H.A. Hassan. Positive solutions of $q$-difference equation. Proc. Amer. Math. Soc., 138:1733-1738, 2010.

http://dx.doi.org/10.1090/S0002-9939-09-10185-5.

[21] R. Finkelstein. The q-Coulomb problem. J. Math. Phys., 37:2628-2636, 1996. http://dx.doi.org/10.1063/1.531532.

[22] R. Finkelstein and E. Marcus. Transformation theory of the q-oscillator. $J$. Math. Phys., 36:2652-2672, 1995. http://dx.doi.org/10.1063/1.531057.

[23] R. Floreanini and L. Vinet. Automorphisms of the $q$-oscillator algebra and basic orthogonal polynomials. Phys. Lett. A, 180:393-401, 1993. http://dx.doi.org/10.1016/0375-9601(93)90289-C.

[24] R. Floreanini and L. Vinet. Symmetries of the $q$-difference heat equation. Lett. Math. Phys., 32:37-44, 1994. http://dx.doi.org/10.1007/BF00761122.

[25] R. Floreanini and L. Vinet. Quantum symmetries of $q$-difference equations. $J$. Math. Phys., 36:3134-3156, 1995. http://dx.doi.org/10.1063/1.531017. 
[26] P.G.O. Freund and A.V. Zabrodin. The spectral problem for the $q$-KnizhnikZamolodchikov equation and continuous q-Jacobi polynomials. Comm. Math. Phys., 173:17-42, 1995. http://dx.doi.org/10.1007/BF02100180.

[27] G. Gasper and M. Rahman. Basic Hypergeometric Series. Cambridge University Press, Cambridge, 1990.

[28] S. Heikkila and V. Lakshmikantham. Monotone Iterative Technique for Nonlinear Discontinuous Differential Equations. Marcel Dekker Inc., New York, 1994.

[29] S. Hu and N.S. Papageorgiou. Handbook of Multivalued Analysis, vol. I: Theory. Kluwer, Dordrecht, 1997.

[30] S. Hu and N.S. Papageorgiou. Handbook of Multivalued Analysis, vol. II: Applications. Kluwer, Dordrecht, 2000.

[31] M.E.H. Ismail and P. Simeonov. $q$-Difference operators for orthogonal polynomials. J. Comput. Appl. Math., 233:749-761, 2009. http://dx.doi.org/10.1016/j.cam.2009.02.044.

[32] F.H. Jackson. On q-difference equations. Amer. J. Math., 32:305-314, 1910. http://dx.doi.org/10.2307/2370183.

[33] F. Jarad, T. Abdeljawad and D. Baleanu. Stability of q-fractional nonautonomous systems. Nonlinear Anal. Real World Appl., 14:780-784, 2013. http://dx.doi.org/10.1016/j.nonrwa.2012.08.001.

[34] V. Kac and P. Cheung. Quantum Calculus. Springer, New York, 2002.

[35] M. Kisielewicz. Differential Inclusions and Optimal Control. Kluwer, Dordrecht, The Netherlands, 1991.

[36] W.-S. Li, J.J. Nieto and Y.-K. Chang. On impulsive hyperbolic differential inclusions with nonlocal initial conditions. J. Optim. Theory Appl., 140:431442, 2009. http://dx.doi.org/10.1007/s10957-008-9468-1.

[37] W.-S. Li, J.J. Nieto and Y.-K. Chang. Solvability of impulsive neutral evolution differential inclusions with state-dependent delay. Math. Comput. Modelling, 49:1920-1927, 2009. http://dx.doi.org/10.1016/j.mcm.2008.12.010.

[38] M. Martelli. Rothe's type theorem for non compact acyclic-valued maps. Boll. Math. Ital., 4:70-76, 1975.

[39] T.E. Mason. On properties of the solutions of linear $q$-difference equations with entire function coefficients. Amer. J. Math., 37:439-444, 1915. http://dx.doi.org/10.2307/2370216.

[40] J.J. Nieto. An abstract monotone iterative technique. Nonlinear Anal., 28:19231933, 1997. http://dx.doi.org/10.1016/S0362-546X(97)89710-6.

[41] G.V. Smirnov. Introduction to the Theory of Differential Inclusions. American Mathematical Society, Providence, RI, 2002.

[42] A. Tarski. A lattice-theoretical fixpoint theorem and its applications. Pacific J. Math., 5:285-309, 1955. 